\title{
Method for Prediction of Acute Hypotensive Episodes
}

\author{
Vytautas Petkus $^{1}$, Alfonsas Vainoras ${ }^{1,2}$, Kristina Berskiene ${ }^{1,2}$, Zenonas Navickas ${ }^{1}$, \\ Rimtautas Ruseckas ${ }^{2}$, Ian Piper ${ }^{3}$, Mantas Deimantavicius ${ }^{1}$, Arminas Ragauskas ${ }^{1}$ \\ ${ }^{1}$ Health Telematics Science Institute, Kaunas University of Technology, \\ Barsausko St. 59 A553-A561, LT-51423 Kaunas, Lithuania \\ ${ }^{2}$ Sports Institute, Medical Academy, Lithuanian University of Health Sciences \\ Tilzes St. 18, LT-47181 Kaunas, Lithuania \\ ${ }^{3}$ Clinical Physics, Southern General Hospital, Glasgow, Scotland,UK \\ telematics@ktu.lt
}

\begin{abstract}
Hypotension is type of secondary insult and it is related to poor outcome. The ability to predict adverse hypotensive events, where a patient's arterial blood pressure drops to abnormally low levels, would be of major benefit to the fields of primary and secondary health care. The aim of the paper is to present the novel method for predicting of acute hypotensive episodes, based on ECG analysis by the complex system theory approach. 45 patients (in four neurointensive care facilities throughout Europe) data were selected for the analysis. 11 patients had EUSIG-defined hypotensive events. The method includes determining of time varying biomarkers corresponding to plurality of physiological processes in patient's organism as a non-linear dynamic complex system and generating an acute hypotension prediction classifier. The calculations of biomarkers are based on complex system approach and algebraic matrix analysis of ECG parameters. The classifier is based on the comparison of biomarkers behaviour in 3D images. It is demonstrated that the presented method allows us to predict arterial hypotension events 40-50 minutes ahead with a sensitivity of $81 \%$, specificity $94 \%$. This result was obtained from prospective real-time data collection in a live clinical intensive care environment.
\end{abstract}

Index Terms-Acute Hypotensive episodes, ECG, complex systems.

\section{INTRODUCTION}

Patients being treated in an intensive care unit for traumatic brain injury are at risk of secondary insults [1], [2]. These insults can lead to further injury to the brain by potentiating pathological processes in the tissue. The secondary insults can be both intracranial and systemic. The intracranial insults predominantly result from high intracranial pressure caused by intracranial hematomas and swelling of the brain. One of the most dangerous types of systemic secondary insults is hypotension, critically low arterial blood pressure. Hypotension is one type of

Manuscript received 12 May, 2015; accepted 15 November, 2015.

This research was funded by a grant (No. VP1-3.1-ŠMM-10-V-02-003) from the Ministry of Education and Science Lithuania. This research was performed by Health Telematics Science Institute at Kaunas University of Technology. Clinical data were collected in Institute of Cardiology of Lithuanian University of Health Sciences, Vilnius University Hospital Santariskiu Klinikos and Republican Vilnius University Hospital. secondary insult and has been shown to be related to poor outcome [1], [3]. The ability to predict adverse hypotensive events, where a patient's arterial blood pressure drops to abnormally low (and dangerous) levels, would be of major benefit to the fields of primary and secondary health care, and especially to the traumatic brain injury domain [4].

Recently, the rapid development of counting techniques and technology make it possible to collect increasing amounts of information and perform modern data analysis. On the other hand, the growth of medical knowledge and supply of various means such as diagnostic devices, advanced electronic tools for heterogeneous signals and for data storage create difficulty in carrying out the analysis of data summaries that are adequate for volume of information collected. Over the past decade, the non-linear dynamics methods based on deterministic chaos and the complex system theories to analyze the complex physiological systems have been introduced [5]. Till the second half of the twentieth century the empirical, phenomenological or statistical methodology dominated, and the end of the twentieth century led to the formation of the chaos and complex system theory. The traditional methods based on self-regulation and statistics appear to be insufficient for the analysis of non-linear and non-stationary signals generated by the living organism. The main feature of the physiological systems - their complexity "hidden" in the biomedical signals. The processing of these signals in terms of complex systems provides the possibilities to perceive the system components and dynamic interrelationships [6], [7]. The breakthrough in the complex system theory broadened the area of its application - from the molecular to the body levels. The basic cardiovascular research, widely used in clinical practice is an electrocardiogram (ECG) recording. The new electronic technologies allowed automatically to record the characteristic points of ECG signal during each cardiocycle time, and to use them for examining of the different dynamic processes on different time scales.

Moreover, it provided a selection of combinations of the parameters that reflected best different processes occurring in the heart and the detailed analysis of the level of these processes. Small and fast changes occurring may have 
significant consequences. Thus, it gives an opportunity to assess the synergies of interacting systems, all system complexity and adaptation [8].

A wealth of data exist in health care systems providing information on the major health indicators of patients in hospitals (blood pressure, temperature, ECG, EEG, etc.), if enough of these data could be drawn together and analysed in a systematic way, then a system could be built that will trigger an alarm predicting the onset of a hypotensive event over a useful time scale [[3]]. However, when the acute hypotensive episodes are not predicted in time, the practitioners are left with insufficient time to determine the causes of the acute hypotensive episodes and to start patient specific treatment. Also, due to insufficient time appropriate treatment may not be administered. If an acute hypotensive episode is not promptly treated, it may result in an irreversible organ damage and, eventually death [9].

In view of these problems, the aim of the paper is to present the method for predicting of acute hypotensive episodes, based on ECG analysis by the complex system theory approach, which embraces holism of the systems analysed, as well as interrelationships of their components.

\section{MATERIALS AND METHODS}

\section{A. Hypotension Definition}

In order to quantify the occurrence of hypotension the Edinburgh University Secondary Insult Grading (EUSIG) system was used, which defines hypotension as systolic arterial pressure (BPs) $\leq 90 \mathrm{mmHg}$ or mean arterial pressure $(\mathrm{BPm}) \leq 70 \mathrm{mmHg}$ sustained for at least 5 minutes. This definition has been shown to be a clinically relevant measure when assessing the burden of hypotension [3]. For the method for predicting a hypotensive event over a useful time scale we have required at least 90 min prehistory with no hypotensive events

\section{B. Patient Material}

45 patients were selected in four neurointensive care facilities throughout Europe (Kaunas, two in Vilnius (Lithuania) and Glasgow (Scotland)). For all patients arterial blood pressure was registered minute by minute and ECG recorded continuously. The criteria for including patients into analysis were: at least $90 \mathrm{~min}$ of artefact-free BP and ECG data records during pre-hypotensive period (for patients with hypotension event) and $120 \mathrm{~min}$ of artefact free data for patients without hypotension event. All patients (11 patients with hypotensive events and 34 patients without hypotensive events) were included in ROC analysis.

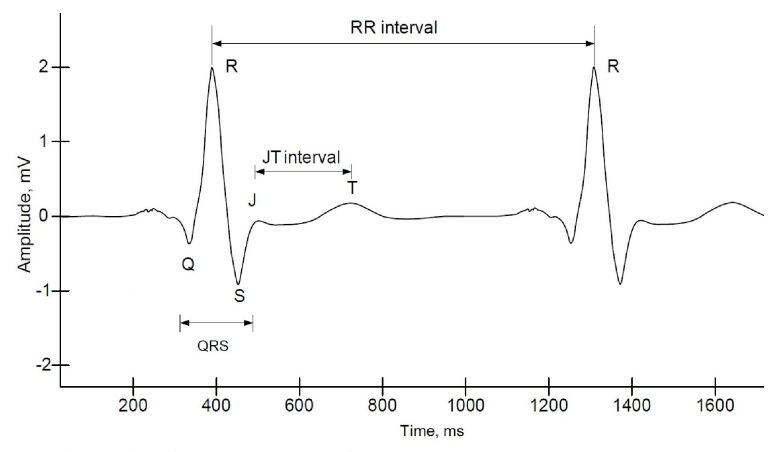

Fig. 1. The main fiducial points of ECG.

\section{The Definition of Diagnostic Biomarkers for Prediction of Hypotensive Events}

The method includes determining of time varying biomarkers corresponding to plurality of physiological processes in patient's organism as a non-linear dynamic complex system and generating an acute hypotension prediction classifier.

The computerized electrocardiographic registration and the analysis system "Kaunas - Load W03" [8], developed at the Institute of Cardiology of Lithuanian University of Health Sciences, were used for evaluating the functional state of the cardiovascular system and for extracting of main fiducial points of ECG signal for each cardio cycle (Fig. 1): $R R$ interval $(\mathrm{ms})$ - it is an interval between two one after another following $\mathrm{R}$ waves. A total body functionality can be described according to RR interval and in particular regulatory processes also it is describing systemic level of the human body; JT interval (ms) - the interval from the ECG junction point $\mathrm{J}$ to the $\mathrm{T}$ wave end, which characterizes duration of ventricular repolarisation; $Q R S$ complex (ms) the duration of this complex illustrates the spread of excitation in the heart, describing the cardiac activity of the inner regulatory processes. Selected ECG parameters represent different fractality (complexity) levels of organism: RR interval and JT interval - systemic, QRS complex describes cardiac intrinsic regulatory process. JT and QRS concatenation represents heart's metabolism and level of heart's management.

The systemic analysis of ECG parameters by the complex system approach is a tremendously ambitious task. It is necessary to take into account the dynamic interface of ECG parameters, which are related with different systemic levels. The classical methods for data sets interrelations correlation analysis, cross-correlation analysis, coherence analysis need a big sets of data. Their results are generalized. The complex system adapts to different conditions, the relations between its elements are shifting. Here is presented a new analytical method for the analysis of dynamical interrelations of two signals, which need only three points of each signal.

The ECG signal is processed for calculation of $R R_{n}{ }_{n}, \mathrm{JT}^{\prime}{ }_{n}$ and $Q R S^{\prime}{ }_{n}$ intervals for use in data arrays for each cardio cycle (n) measured(three time series which elements are determined). For these calculations, $\mathrm{RR}_{\mathrm{n}}{ }^{\text {' interval is used as }}$ a time stamp (marker) for all calculations used during processing and also used for the synchronization of ECG and blood pressure data. Then data are normalized using following formulas

$$
J T_{n}=\frac{J T_{n}^{\prime}-J T_{\min }}{J T_{\max }-J T_{\min }}
$$

where $J T_{\min }=140 \mathrm{~ms}, J T_{\max }=400 \mathrm{~ms}$

$$
r Q R S_{n}=\frac{Q R S_{n}{ }^{\prime}-Q R S_{\min }}{Q R S_{\max }-Q R S_{\min }}
$$

where $Q R S_{\text {min }}=40 \mathrm{~ms}, Q R S_{\max }=150 \mathrm{~ms}, \mathrm{n}=(0,1,2$, etc.$)$ is the number of cardio cycles measured. 
Then a series of second order matrixes $A_{n}$ is constructed as follows

$$
A_{n}=\left[\begin{array}{cc}
J T_{n} & J T_{n-1}-Q R S_{n-1} \\
J T_{n+1}-Q R S_{n+1} & Q R S_{n}
\end{array}\right],
$$

for every cardio cycle $n$. Calculations of mathematical characteristics: difference of matrix $A_{n}$ : $\operatorname{dfr}\left(A_{n}\right):=J T_{n}-Q R S_{n}$, co-diagonal product of matrix $A_{n}$ : $\operatorname{cdp}\left(A_{n}\right):=\left(J T_{n-1}-Q R S_{n-1}\right) \times\left(J T_{n+1}-Q R S_{n+1}\right) . \quad$ The values of discriminates (dsk) as an estimate of concatenation of two sequences and of ECG parameters is calculated as follows

$$
\operatorname{dsk}\left(J T_{n}, Q R S_{n}\right)=\operatorname{dsk}(A n)=\operatorname{dfr}\left(A_{n}\right)^{2}+4 \operatorname{cdp}\left(A_{n}\right) .
$$

If values of discriminates become close to zero, then numeric time series become similar - their concatenation is high - discriminates describe related systems [10]. The slope of linear dependence between $\operatorname{dsk}\left(J T_{n}, Q R S_{n}\right)$ and $J T_{n}$ for each 20 cardio cycles results in $J(\operatorname{dsk}(J T, Q R S), J T)$; and the ratio between the standard deviation and the mean of $\operatorname{dsk}\left(J T_{n}, Q R S_{n}\right)$ in each 20 cardio cycles of $\operatorname{dsk}\left(J T_{n}, Q R S_{n}\right)$ results in $V[\operatorname{dsk}(J T, Q R S)]$. Then input data of $J(\operatorname{dsk}(Q R S, J T))$ and $V(\operatorname{dsk}(Q R S, J T))$ pairs reading and forming of the data array $A\left\{t_{i}, 1 \ldots N\right\}$. Data array $A\left\{t_{i}\right.$, $1 \ldots N\}$ is formed from pairs of $\mathrm{J}(\operatorname{dsk}(Q R S, J T), J T)$ and $V(\operatorname{dsk}(Q R S, J T))$ data points received within a set time interval (preferably $15 \mathrm{~min})$. Approximate number of points of data array $N$ is $\sim 45$ ( $\sim 3$ points per minute). Data are updated periodically every 5 minutes by forming new data array $A\{\quad t, \quad 1 \ldots N\}$. Pairs of $J(\operatorname{dsk}(Q R S, J T) J T)$ and $V(\operatorname{dsk}(Q R S, J T))$ points are plotted in field $J(\mathrm{y}-$ axis $)$ vs $V(\mathrm{x}$ - axis). The field area is limited from $\min \mathrm{V}=-0.5$ to $\max$ $\mathrm{V}=5$ in $\mathrm{x}$ axis. Field area is limited from $\min \mathrm{J}=-4$ to $\max$ $\mathrm{J}=4$ in y axis. Limited area is segmented by steps $\Delta_{\mathrm{V}}=0.25$ in $\mathrm{x}$ axis and $\Delta_{\mathrm{S}}=0.2$ in y axis. Finally, the two dimensional (2D) array of data points distribution density - $D\left\{t_{i}, 1 \ldots n\right.$, $1 \ldots m\}$ is calculated. Next, contour plot is calculated on density $D\left\{t_{i}, 1: n, 1: m\right\}$ on set threshold level $=0.29 *$ $\max \left(D\left\{t_{i}, 1 \ldots n, 1 \ldots m\right\}\right)$. The contour plot also can be calculated from the density function $D\{t, 1: n, 1: m\}$ using different threshold values, e.g. level +0.29 below the maximal value 1.0 of $\max D$ or other levels. Parameters are calculated during each cycle of data processing: area of the contour is Area $t_{i}$ ) (the sum value of all areas is calculated if there are only a few contours found), maximum value of density function $D_{\max }\left(t_{i}\right)=\max \left(D\left\{t_{i}, 1: n, 1: m\right\}\right)$ and normalized value of maximum density $D_{\text {max_NORM }}\left(t_{i}\right)=\max$ $\left(D\left\{t_{i}, 1: n, 1: m\right\}\right) / \operatorname{sum}\left(D\left\{t_{i}, 1: n, 1: m\right\}\right)$. The values of arrays $D_{\text {max_NORM }}\left(t_{i}\right)$ and Area $\left(t_{i}\right)$ are the tested measures for ROC analysis.

\section{RESULTS}

At first for each patient the arterial blood pressure was evaluated by EUSIG-defined hypotensive events definition. If the hypotensive event was found - the start minute of the event was stated. The illustration on mean arterial blood pressure $\mathrm{BPm}$ analysis of one patient is given in Fig. 2(a).

Then the biomarkers from ECG parameters are calculating $J(\operatorname{dsk}(Q R S, J T))$ and $V(\operatorname{dsk}(Q R S, J T))$, measures $D_{\text {max_NORM }}$ and Area are calculated. The 3D images of fluctuations of mentioned biomarkers for the same patient are given in Fig. 3. Affirmative prediction is indicated by comparing two 3D images - initial one peak image and other images with less height than the main peak and with other peaks which represent chaotic process. The 3D image representing the chaotic process reflects the patient is approaching hypotensive event. When the patient is healthy the images will show a simple peak representative of a steady state. As the patient becomes less healthy, begins to depart from a steady state we have more chaotic images. The closer the patient gets to a hypotensive event the more and more chaotic the images become (as the system measurements move further from a steady state) which is indicative of a system when the system is unstable.

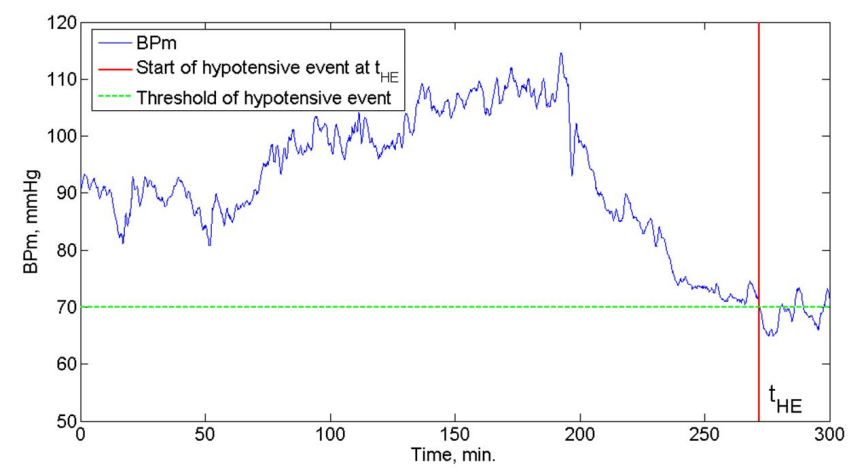

a)

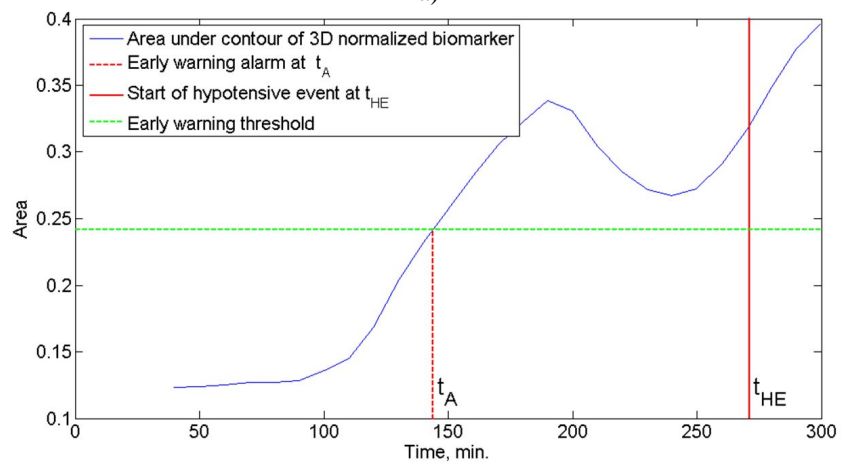

b)

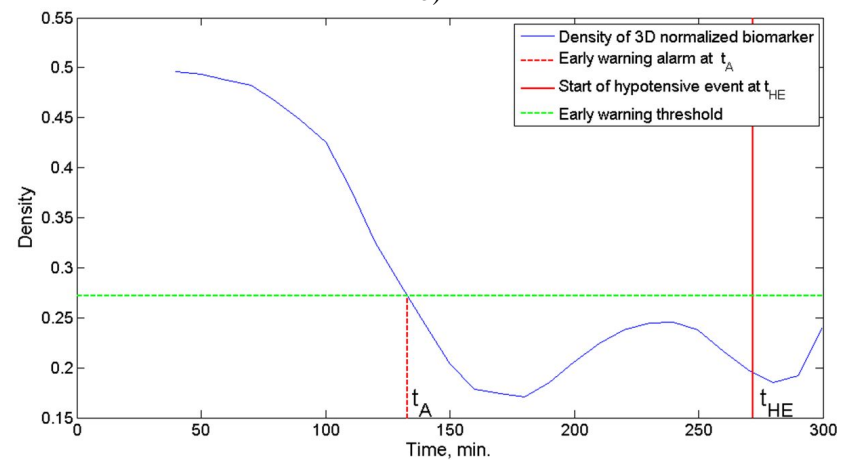

c)

Fig. 2. BPm fluctuations (a) and dynamics of $D_{\text {max_NORM }}$ and Area factors for early detection of hypotensive event: a) - area under curve of 3D normalized biomarker (Area), c) - density of 3D normalized biomarker $\left(D_{\text {max_NORM }}\right)$. Time of hypotensive event is tHE $=271 \mathrm{~min}$. Time of alarm before hypotensive event is $\mathrm{t}_{\mathrm{A}}=144 \mathrm{~min}(\mathrm{~b})$ and $\mathrm{t}_{\mathrm{A}}=133 \mathrm{~min}(\mathrm{c})$. 


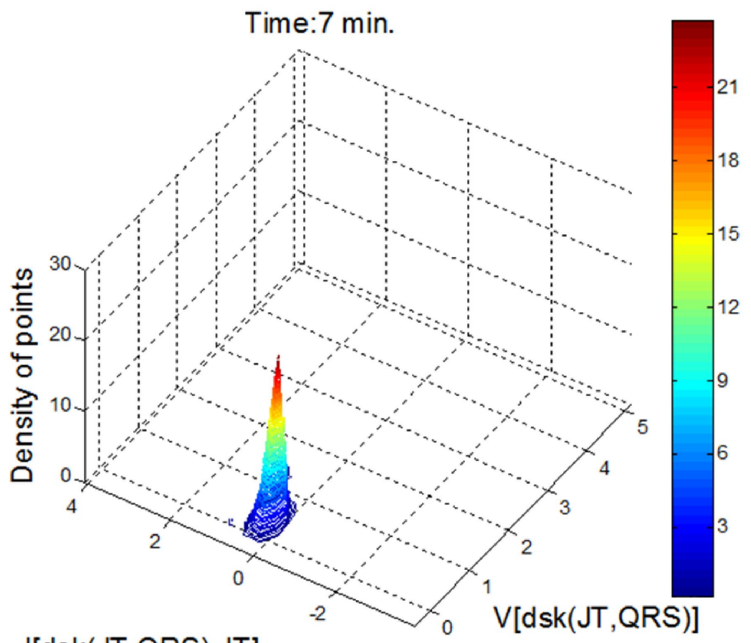

J[dsk(JT,QRS),JT]

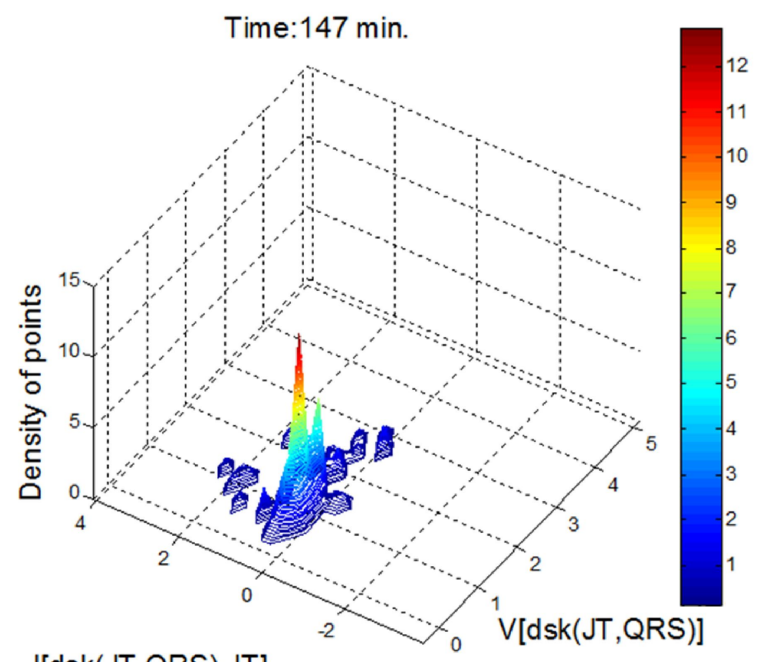

J[dsk(JT,QRS),JT]

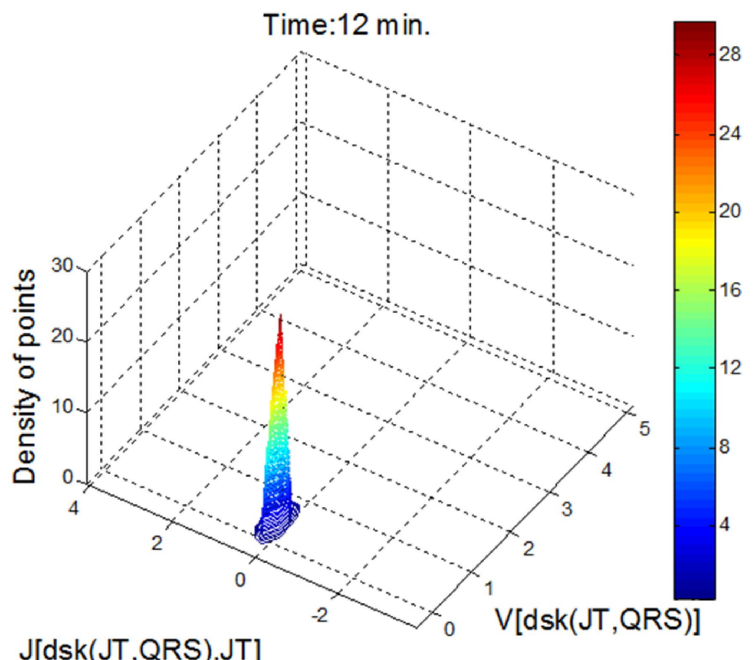

J[dsk(JT,QRS),JT]

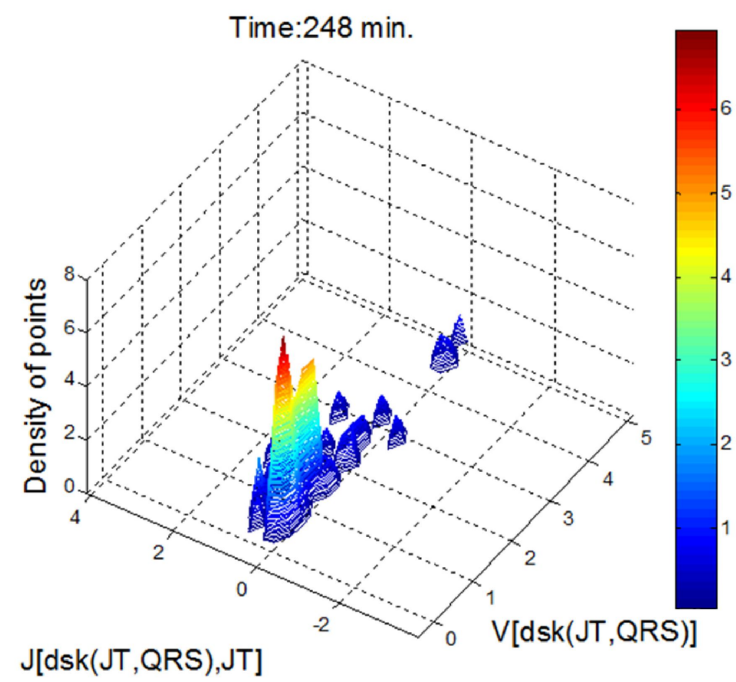

Fig. 3. The dynamics of 3D images of biomarkers at different moments before hypotensive event (271 min.) for the same patient as in Fig. 1.

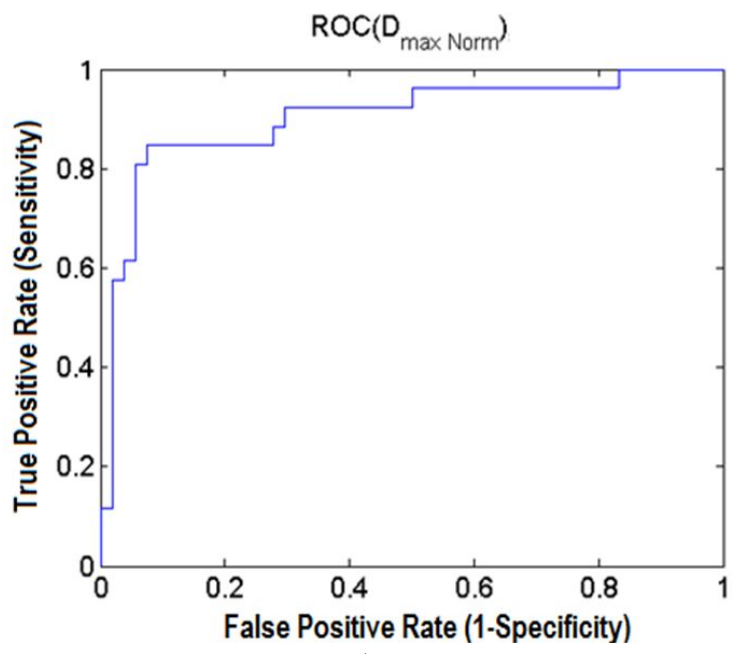

a)

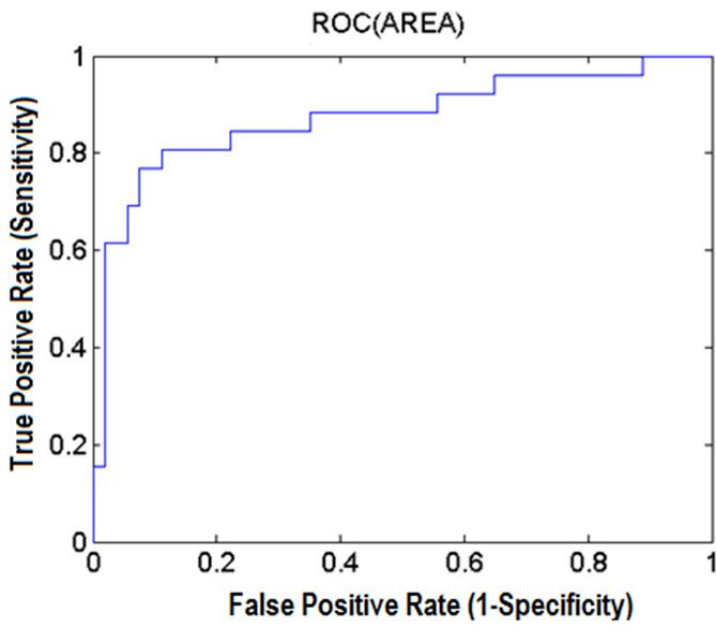

b)

Fig. 4. The ROC curves of $D_{\max \_N O R M}$ (a) and Area (b) for all tested patients.

For the predicting of acute hypotensive episodes $D_{\text {max_NORM }}$ and Area measures were selected (the illustration for the same patient is in Fig. 2(a) and Fig. 2(b)) for the ROC analysis. ROC curves were calculated for all 45 patients by averaging data in 40 minutes interval. The best prognosis was performed 90 minutes before the event (it was evaluated 120, 90 and 60 minutes). We have calculated the sensitivity and specificity: the better results were for the
$D_{\text {max_NORM }}$ (Area under the curve - $90 \%$; sensitivity - $81 \%$; specificity - $94 \%$ ). By using Area we got such evaluations: Area under the curve $-87 \%$; sensitivity $77 \%$; specificity $93 \%$. ROC curves are presented in Fig. 4. Determined common thresholds corresponding to best sensitivity point for early prediction of hypotensive event are 0.242 for Area and 0.272 for $D_{\text {max } \_N O R M}$ factors, i.e. hypotensive event will be alarmed at time moment $t_{A}$ when Area(t) $>0.265$ or 
$D_{\text {max_NORM }}<0.272$ (Fig. 2(a) and Fig. 2(b)). So, the presented method is credible for the prediction of acute hypotensive events.

\section{DISCUSSION}

It is known that the cumulative duration of hypotensive events is a prognostic factor for their clinical outcome in head injury [11].The importance of reducing the burden of hypotension is not restricted to the TBI population; for with the medical management of sepsis, it has been shown that duration of hypotension before initiation of effective antimicrobial therapy is the critical determinant of survival [12]. The ability to pick up even one potentially emerging hypotensive event before it has happened is clinically important. The AVERT-IT technology (based upon a machine learning approach) can predict arterial hypotension events 15-30 minutes ahead with a sensitivity of $37.51 \%$ ), specificity $91.20 \%$ [13]. But the results are unlikely to be sufficient for the direct clinical practise. The presented method produced sensitivity - $81 \%$; specificity - $94 \%, 90$ minutes before the event - it gives the nursing and medical staff more time to assess the patient and potentially prevent the event from occurring, or, to treat it sooner and less aggressively. In the medical management of sepsis, it has been shown that with each hour delay in treatment of hypotension leads to an increase in in-hospital mortality of $7.6 \%$ [12]. Another potential clinical outcome of this work is that fostering earlier and less aggressive intervention will in turn lead to a quantifiable reduction in the duration of average patient stay, with associated reduction in cost of intensive care management (in 2005, typically estimated at c. $£ 1,200 /$ patient/day) [14].

The problem of invalid or noisy data may have caused the incidence of hypotension to be underestimated in this study also the elimination of the patients. There is no doubt that the technology could be improved further by research including more ECG parameters and their concatenations, also synchronous registered EEG data. The prediction would be better if we consider to patients sex, age, diagnosis. Also the method should be tested with a representative sample size and finally to assess the robustness and clinical utility of this new approach, the model should be re-evaluated prospectively in a live clinical environment with streaming ECG data being fed from patients monitored across different centres.

\section{CONCLUSIONS}

The presented method has been shown to predict arterial hypotension events 40-50 minutes ahead with a sensitivity of $81 \%$, specificity $94 \%$. This result has been obtained from real-time data collection in a live clinical environment.

Two areas of further research are warranted: a) Research towards improving detection and removal of artifact from ECG and BP data and b) Research on optimising this method for the displaying of hypotensive warnings to medical staff using prediction data.

\section{REFERENCES}

[1] R. Donald, T. Howells, I. Piper, I. Chambers, G. Citerio, P. Enblad et al., "Trigger characteristics of EUSIG-defined hypotensive events", Acta Neurochir Suppl, vol. 114, pp. 45-49, 2012. PMID: 22327663. [Online]. Available: http://dx.doi.org/10.1007/978-3-7091-0956-4_9

[2] J. D. Miller, D. B. Becker, "Secondary insults to the injured brain", $J$ $R$ Coll Surg Edinb, vol. 27, pp. 292-298, 1982.

[3] P. A. Jones, P. J. Andrews, S. Midgley, S .I. Anderson, I. R. Piper, J. L. Tocher, et al., "Measuring the burden of secondary insults in head-injured patients during intensive care", J Neurosurg Anesthesiol, vol. 6, pp. 4-14, 1994. [Online]. Available: http://dx.doi.org/ 10.1097/00008506-199401000-00001

[4] A. Stell, R. Sinnott, J. Jiang, R. Donald, I. Chambers, G. Citerio, et al., "Federating distributed clinical data for the prediction of adverse hypotensive events", Philos Trans A Math Phys Eng Sci, vol. 367, pp. 2679-2690, 2009. [Online]. Available: http://dx.doi.org/ 10.1098/rsta.2009.0042

[5] P. Erdi, Complexity Explained. Springer, 2008, p. 390.

[6] T. S. Deisboec, J. Y. Kresh, Complex Systems Science in Biomedicine. Topics in Biomedical Engineering: International Book Series. Springer, 2006, p. 864. [Online]. Available: http://dx.doi.org/10.1007/978-0-387-33532-2

[7] A. Quarteroni, L. Formaggia, A. Veneziani, Complex Systems in Biomedicine. Springer, 2006, p. 292. [Online]. Available: http://dx.doi.org/10.1007/88-470-0396-2

[8] K. Berskiene, A. Lukosevicius, G. Jarusevicius, V. Jurkonis, Z. Navickas, A. Vainoras, et al. "Analysis of dynamical interrelations of electrocardiogram parameters", Elektronika ir Elektrotechnika, vol. 7, no. 95, pp. 95-98, 2009.

[9] R. Donald, T. Howells, I. Piper, I. Chambers, G. Citerio, P. Enblad, et al., "Early warning of EUSIG-defined hypotensive events using a Bayesian Artificial Neural Network", Acta Neurochir Suppl, vol. 114, pp. 39-44, 2012. [Online]. Available: http://dx.doi.org/10.1007/9783-7091-0956-4_8

[10] Z. Navickas, L. Bikulciene, "Expressions of solutions of ordinary differential equations by standard functions", Mathematical Modeling and Analysis, vol. 11, pp. 399-412, 2006.

[11] A. Marmarou, R. L. Anderson, J. D. Ward JD, S. C. Choi, H. F. Young, H. M. Eisenberg, et al., "Impact of ICP instability and hypotension on outcome in patients with severe head trauma", Journal of Neurosurgery, vol. 75, no. 1S, pp. 59-66, 1991. [Online]. Available: http://jnsonline.org/jns/issues/v75n5s/pdf/n0750059.pdf

[12] A. Kumar, D. Roberts, K. E. Wood, B. Light, J. E. Parrillo, S. Sharma et al., "Duration of hypotension before initiation of effective antimicrobial therapy is the critical determinant of survival in human septic shock", Critical Care Medicine, vol. 34, no. 6, pp. 1589-1596, 2006. [Online]. Available: http://dx.doi.org/10.1097/01.CCM. 0000217961.75225.E9

[13] EC FP7 Avert-IT Project - Advanced Arterial Hypotension Adverse Event Prediction through a Novel Bayesian Neural Network. [Online]. Available: ttp://cordis.europa.eu/project/rcn/85474_en.html

[14] J. F. Dasta, T. P. McLaughlin, S. H. Mody, C. T. Piech, "Daily cost of an intensive care unit day: The contribution of mechanical ventilation", Critical Care Medicine, vol. 33, no. 6, pp. 1266-1271, 2005. [Online]. Available: http://dx.doi.org/10.1097/01.CCM. 0000164543.14619 .00 